\title{
Influência das Funções Executivas Sobre a Marcha e o Equilíbrio de Idosas Praticantes Regulares de Exercícios Físicos
}

\section{The Influence of Executive Functions on Gaitand Balance among Elderly People Regularly Practicing Physical Exercise}

\author{
MARCELO DE MAIO NASCIMENTO ${ }^{1}$ \\ NAYRA JÉSSICA DOS SANTOS MAIA ${ }^{2}$ \\ LUCAS DA SILVA RAMOS ${ }^{2}$ \\ HANS JOACHIM APPELL ${ }^{3}$
}

\section{RESUMO}

Objetivos: examinar as funções executiva (FE) de idosas fisicamente ativas, determinando sua influência sobre o desempenho em testes de marcha e equilíbrio corporal. Material e Métodos: Trata-se de um estudo observacional exploratório com delineamento transversal. Participaram 74 indivíduos do sexo feminino $(66,80 \pm 6,73$ anos), praticantes regulares de exercícios físicos. Os instrumentos utilizados foram um questionário para incidência de comorbidades, o Miniexame do Estado Mental de Saúde (MEEM), Teste de Fluência Verbal (FV), teste Time Up and Go, versão simples (TUGs), versão motora (TUGm) e versão cognitiva (TUGc), Dynamic Gait Index (DGI) e o Teste de Equilíbrio Corporal (TEC). Resultados: A análise dos dados demonstrou influência das FE sobre o processamento neural, necessário aos ajustes do controle da marcha e do equilíbrio corporal. Da mesma forma, que o déficit nas FE aumenta a probabilidade de queda de mulheres idosas praticantes regulares de exercícios físicos, especialmente, aquelas com baixa escolaridade e em idade superior a 75 anos. A análise de regressão linear múltipla explicou a probabilidade de queda causada pela interferência do déficit cognitivo sobre os testes TUGs (R2 $=0,497, p<0,001)$, TUGm (R2=0,479, $p<0,001)$, TUGc $(R 2=0,405, p<0,001), D G I(R 2=0,445$, $p<0,001)$ e TEC (R2=0,444, $p<0,001)$. Conclusão: Concluiuse que o déficit cognitivo se apresenta como fator de risco de queda para mulheres praticantes regulares de exercícios físicos.

\section{DESCRITORES}

Quedas.Equilíbrio Postural.Cognição.Idoso.

\begin{abstract}
Objective: To examine the influence of executive functions (EF) on the performance of gait and body balance tests among elderly people regularly practicing physical exercise. Material and Methods: This was an exploratory observational study with a cross-sectional design. The sample was composed of 74 female subjects ( $66.80 \pm 6.73$ years), who were regular physical exercise practitioners. The instruments used for data collection were, as follows: a questionnaire for the incidence of comorbidities, Mental Health Status Mini-test (MMSE), Verbal Fluency Test (VF), Time Up and Go test, simple version (TUGs), motor version (TUGm) and cognitive version (TUGc), Dynamic Gait Index (DGI) and Body Balance Test (TEC). Results: Data analysis revealed the influence of EF on neural processing, necessary for gait control and body balance adjustments. Likewise, the deficit in the EF increases the probability of falling among elderly women practicing regular physical exercises, especially those with low schooling and over 75 years old. Multiple linear regression analysis explained the probability of falling due to cognitive deficit interference on TUGs (R2 $=0.467, p<0.001)$, TUGm $(R 2=0.479, p<0.001)$, TUGc $(R 2=0.405, p<0.001), D G I$ $(R 2=0.445, p<0.001)$ and TEC $(R 2=0.444, p<0.001)$. Conclusion: Cognitive deficit presents as a risk factor for falls among women regularly practicing physical exercises.
\end{abstract}

\section{DESCRIPTORS}

Falls.Cognition.Postural Balance.Elderly.

\footnotetext{
Professor do curso de Educação Física da Universidade Federal do Vale do São Francisco (UNIVASF). Petrolina. Pernambuco. Brasil.

2 Discente do curso de Medicina da Universidade Federal do Vale do São Francisco (UNIVASF). Petrolina. Pernambuco. Brasil.

3 Coordenador do Instituto de Anatomia e Fisiologia da Universidade de Esportes da cidade de Colónia, (Die Deutsche Sporthochschule Köln-SPOHO). Colónia. Alemanha.
} 
$m$ se tratando da população idosa, o tema quedas é considerado como uma questão de saúde pública ${ }^{1}$. Estudos demonstraram que um terço dos idosos em idade igual ou superior à 65 anos já experimentam uma queda ${ }^{2,3}$, além de que a incidência aumenta em $50 \%$ junto à população feminina ${ }^{4,3}$. Assim, sabendo-se que a população idosa brasileira cresceu, consideravelmente, nas últimas décadas e que as projeções indiquem que o Brasil terá no ano de 2060 cerca de 58,4 milhões de habitantes idosos, o tema queda merece atenção e estudo 5 .

Em idade avançada, a queda e sua recorrência têm influência direta sobre a percepção da qualidade de vida (QV), visto que são responsáveis por lesões, dias de hospitalização, conduzindo, em certos casos, o indivíduo até mesmo à morte ${ }^{2,6}$. Paralelo ao fato, há de se considerar que quedas acarretam custos aos cofres públicos ${ }^{3}$. Suas causas podem sobrevir de fatores extrínsecos e intrínsecos, assim como da interação entre ambos ${ }^{1}$. Fatores extrínsecos estão relacionados ao meio ambiente, consistindo em obstáculos dispostos no espaço ou decorrentes de problemas de iluminação. Já fatores intrínsecos consistem em aspectos próprios da pessoa, muitos relacionados ao processo do envelhecimento humano ${ }^{7,8}$. Dentre eles háo déficit das funções executivas (FE).

As FE são definidas como um conjunto de habilidades cognitivas necessárias para planejar, executar, sequenciar e monitorar ações complexas dirigidas para um determinado fim ${ }^{9,10}$. Alterações nessas capacidades podem interferir consideravelmente sobre o desempenho da marcha ${ }^{11,12}$ edo equilíbrio estático e dinâmico ${ }^{8,12,13}$. O caso ganha ênfase em situações de tarefa dupla ou múltipla ${ }^{14,15}$, quando o indivíduo deve resolver duas ou mais tarefas ao mesmo tempo, o que, automaticamente, exige sua atenção e concentração. Em consequência disso, o termo FE tem sido amplamente utilizado para designar diferentes ações de ordem cognitiva, como concentração, atenção, planejamento, grau de abstração, seletividade de estímulos, flexibilidade de controle mental e memória operacional ${ }^{16}$. Admite-se que tanto a velocidade cognitiva, como o potencial estratégico da pessoa à construção de categorias fonéticas incidem em propriedades das FE.

$O$ caso pode ser investigado por meio do teste de fluência verbal $(F V)^{16,17}$, o qual analisa a aptidão da pessoa para criar palavras, proceder trocas e compor agrupamentos, em curto espaço de tempo ${ }^{18}$. O desempenho neste teste possibilita diagnosticar a habilidade cognitiva da pessoa ${ }^{16,19}$. Logo comprometimentos no teste de FV sugerem déficit no desempenho das FE, existindo assim a indicação de algum transtorno à nível cognitivo. Segundo estudos, déficits nas FE exercem influência negativas obre o padrão da marcha ${ }^{10,20} \mathrm{e}$ controle do equilíbriocorporal ${ }^{15}$, o que, por conseguinte, aumenta o risco de queda. Isso significa dizer que a avaliação do desempenho de idosos em testes de FE incide em medida à predição de quedas ${ }^{8,12}$.

Em se tratando do tema quedas, um procedimento útil para ampliar ou manter a habilidade cognitiva de idosos, consiste na prática regular do exercício físico (EF). A medida se apresenta como uma estratégia de simples execução e baixo custo ${ }^{21}$, muito adotada em programas de prevenção de quedas ${ }^{14}$. Por intermédio do EF há a ativação da circulação cerebral que altera a síntese de neurotransmissores, beneficiando o processamento cognitivo, o qual se torna mais rápido e eficiente. Transformações a nível cognitivo, sobrevindas do exercício físico ocorrem tanto na região do córtex cerebral, como no hipocampo, o qual com o EF sofre aumento de volume ${ }^{21}$. No caso da pessoa idosa, o EF contribui significativamente para o desenvolvimento e/ou manutenção da capacidade de memória e atenção ${ }^{15}$. Nessa perspectiva, sabese ainda que paralelo à melhora do desempenho cognitivo, a prática regular de exercícios físicos também qualificao desempenho do equilíbrio estático e dinâmico e da marcha de indivíduos idosos, aprimorando o comprimento, cadência e velocidade da passada ${ }^{15,22}$.

Diante do exposto, o presente estudo teve por fim, examinar o desempenho das FE de um grupo de mulheres idosas praticantes regulares de 
exercícios físicos, verificando, igualmente, a influência do déficit das FE sobre o desempenho em testes de marcha e equilíbrio corporal.

\section{MATERIAL E MÉTODOS}

Trata-se de um estudo observacional exploratório com delineamento transversal. 0 cálculo amostral foi realizado considerando 100 idosas participantes do programa de atividade física oferecido à comunidade da cidade de Petrolina-PE, pelo curso de Educação Física da Universidade Federal do Vale do São Francisco (UNIVASF). A margem de erro adotada foi de $5 \%$, com prevalência de desfechos de $50 \%$ e nível de confiança de $95 \%$, o que resultou em 72 indivíduos. Contudo considerando que todas as alunas do programa foram convidadas a participarem do estudo, a mostra final foi constituída por 76 indivíduos, todos do sexo feminino $(66,80 \pm 6,73$ anos). A frequência da prática do $E F$ foi de duas vezes por semana (50 minutos), em um dos seguintes grupos: hidroginástica, natação, ginástica geral, Pilates, musculação, tênis. Como critério de inclusão foi adotado a idade e"60 anos, o tempo mínimo de seis meses de prática do EF e $75 \%$ de frequência nas atividades, não apresentar lesão muscular, articular ou óssea no período da avaliação. Foram excluídos indivíduos que não completaram todas as etapas ou não assinaram o Termo de Consentimento Livre e Esclarecido (TCLE). O estudo foi aprovado pelo Comitê de Ética em Pesquisa com Seres Humanos da Universidade Federal do Vale do São Francisco/ UNIVASF (CAAE: 44113715.3.0000.5196).

Dados sociodemográficos e comorbidades foram coletados por intermédio de um questionário específico. Dois testes foram aplicados para o exame do desempenho das FE: o Miniexame do Estado Mental (MEEM) $)^{23}$ o o teste de Fluência Verbal $(\mathrm{FV})^{16,17,18}$. Com o MEEM foram rastreados possíveis casos de demência, os escores dotados foram: 20 pontos para idosas analfabetas, 24 pontos para aquelas com um a quatro anos de escolaridade $e$ 26,5 pontos com escolaridade de cinco anos ou mais ${ }^{23}$.Com o teste de FV foram obtidas informações detalhadas sobre a capacidade de armazenamento do sistema de memória semântica, além da capacidade das participantes para criar em estratégias, organizar, processar e recuperar informações armazenadas na memória de longo tempo. O procedimento consistiu em solicitar às entrevistadas que evocassem o maior número de nomes de animais, no espaço de um minuto. A avaliação do teste de FV ocorreu em intervalos de 15 segundos, observando-se a velocidade do entrevistado para formar agrupamentos, categorias e realizar trocas. A análise do teste de fluência semântica foi realizada mediante categorização de animais, transcorrendo da seguinte forma: i) número total de nomes de animais gerados no tempo de um minuto; ii) quantitativo de nomes de animais gerados a cada 15 segundos; iii) número de categorias, isso significa dizer, a evocação de nomes de animais que representassem uma determinada subcategoria semântica;iv) quantidade de agrupamentos realizados; e, v) quantitativo de trocas.

O desempenho da marcha foi avaliado por três formas do teste Time Up and Go (TUG) ${ }^{24}$ : i) TUG simples (TUGs)que consistiu em solicitar ao participante sentar e se erguer de uma cadeira de $45 \mathrm{~cm}$ de altura, com apoio para os braços, a seguir, caminhar 3 metros, realizar um giro de $180^{\circ} \mathrm{em}$ um cone, retornar à cadeira e sentar; ii) TUG motor $(T U G m)^{24}$,neste, durante a marcha, as participantes transportaram uma bandeja de papelão com $25 \mathrm{~cm}$ de raio, sobre ela havia um copo de plástico vazio com $12 \mathrm{~cm}$ de altura; iii) TUG cognitivo (TUGc) ${ }^{24}$ que consistiuna contagem em voz alta, durante a marcha, em ordem decrescente, iniciando no número 100 (cem).A avaliação do desempenho foiconsiderada normal, sem risco de queda, quando a resolução de tarefas requereu tempo d"10 segundos, a independência parcialmente preservada foi determinada pelo escore entre 11 e 20 segundose a independência funcional comprometida mediante resultados e"20 segundos ${ }^{24}$.

O instrumento Dynamic Gait Index (DGI) ${ }^{25}$ foi aplicado para o exame dealterações da marcha, durante a resolução de tarefas funcionais. O DGI possui oito tarefas de deambulação: marchar em superfície plana, alteração na velocidade 
da marcha, marchar com rotação horizontal de cabeça, marchar com rotação vertical da cabeça,marchar e girar $\left(180^{\circ}\right)$ passo sobre um obstáculo,contornar um obstáculo e subir/ descer degraus.Seu escore máximo é de vinte e quatro pontos. Em idosos com idade e"60 anos, a interpretação do valor d"19 pontos, indicou o risco de queda.

O desempenho do equilíbrio estático e dinâmico foi determinado pelo Teste Equilíbrio Corporal (TEC) ${ }^{26}$. O TEC possui catorze tarefas dispostas em ordem crescente de dificuldade, sete itens avaliam o equilíbrio estático e sete o dinâmico. Oito tarefas são realizadas no solo, seis sobre uma trave de madeira com 4 metros de comprimento, $10 \mathrm{~cm}$ de largura e $3 \mathrm{~cm}$ de altura. Sob a trave são avaliadas estratégias de reação do tornozelo, o passo para trás, giros de $180^{\circ}$ e $360^{\circ}$, além do equilíbrio associado ao objeto. Este instrumento também avalia de forma diferenciada a regulação exteroceptiva (olhos abertos) e interoceptiva (olhos fechados) do equilíbrio ${ }^{26}$. Sua pontuação é dicotômica variando, entre zero e um, com escore máximo de 14 pontos. A interpretação dos resultados permite a diferenciação do desempenho por faixas etárias e gênero (Quadro 1$)^{26}$
A normalidade dos dados foi estabelecida pelo teste de "Komolgorov-Smirnov", seguido pela estatística descritiva (média e desvio padrão). Dados categóricos foram analisados pelo teste Qui Quadrado. O teste de Kruskal Wallis foi utilizado para determinar a significância dos desempenhos nas avaliações, as diferenças intergrupos do exame das $\mathrm{FE}$, marcha e equilíbrio foi determinado pelo teste de Mann-Whitneys, seguido do post hoc de Benferroni. Os efeitos foram testados no nível de 0,0167 de significância. A associação entre as variáveis foi estabelecida pelo coeficiente de correlação de Spearman. Por fim, considerando que o risco de queda, em idosos praticantes regulares de exercícios físicos, não pode ser explicado por um único agente causal,optou-se pelo desenvolvimento da análise de regressão linear múltipla forward. Os dados foram processados no software Statistical Packagefor the Social Sciences (SPSS), versão 23.0.

\section{RESULTADOS}

As principais características sociodemográficas, bem como a incidência de quedas e o consumo de medicamentos das participantes são apresentadas, segundo faixas etárias, na Tabela 1.

\begin{tabular}{|l|c|c|c|c|c|}
\hline $\begin{array}{l}\text { Classificação } \\
\text { Muito Bom }\end{array}$ & $\begin{array}{r}\mathbf{3 0 - 3 9} \\
\text { anos }\end{array}$ & $\begin{array}{c}\mathbf{4 0 - 4 9} \\
\text { anos }\end{array}$ & $\mathbf{5 0 - 5 9}$ anos & $\mathbf{6 0 - 6 9}$ anos & $\begin{array}{r}\mathbf{7 0 - 7 9} \\
\text { anos }\end{array}$ \\
\hline $\begin{array}{l}\text { Homens } \\
\text { Mulheres }\end{array}$ & $>11$ & $>10$ & $>8$ & 8 & 7 \\
\hline Bom & $>10$ & $>10$ & $>8$ & 7 & 6 \\
\hline Homens & $10-11$ & $9-10$ & 8 & $6-7$ & $5-6$ \\
\hline Mulheres & $9-10$ & $9-10$ & $7-8$ & $5-6$ & $4-5$ \\
\hline Reqular & & & & & \\
\hline Homens & $8-9$ & $7-8$ & $6-7$ & $5-6$ & $3-4$ \\
\hline Mulheres & $7-8$ & $7-8$ & $5-6$ & $4-5$ & $4-5$ \\
\hline Fraco & & & & & \\
\hline Homens & $6-7$ & $5-6$ & $4-5$ & $3-4$ & $2-3$ \\
\hline Mulheres & $5-6$ & $5-6$ & $3-4$ & $2-3$ & $1-2$ \\
\hline Muito Fraco & & & & & \\
\hline Homens & $<6$ & $<5$ & $<4$ & $<3$ & $<2$ \\
\hline Mulheres & $<5$ & $<5$ & $<3$ & $<2$ & $<1$ \\
\hline
\end{tabular}

Fonte: Adaptado de Nascimento, Coriolano Appell e Appell Coriolano ${ }^{26}$. 
Observa-se quesexagenárias apresentaram níveis de escolaridade superior as septuagenárias. De acordo com os critérios da $\mathrm{OMS}^{27}$ para o IMC, com exceção das idosas acima de 75 anos, todas as demais foram classificadas na faixa limite do sobrepeso, muito próximo da obesidade, indicando risco desaúde. Não foram observadas interações medicamentosas, visto que a média do consumo diário de medicamentos foi inferior a quatro tipos. Conforme o autorrelato de quedas, constatou-se incidência inferior a dois eventos por pessoa, até 12 meses, antes da realização deste estudo.

A análise estatística demonstrou queda do desempenho,com o avanço da idade, em todas as avaliações (Tabela 1).O teste $U$ Mann Whitney apontou diferenças estatisticamente significativas para o MEEM $(p=0,016)$ entre os grupos de sexagenárias e idosos acima dos 75 anos. Com exceção da FV-Categoria $(p=0,014)$ não foi observada qualquer diferença significativa intergrupos. Seguindo a ordem dos resultados, os testes de mobilidade TUGs, TUGm e DGI indicaram diferença significativa entre idosas sexagenárias e septuagenárias acima dos 75 anos. $O$ desempenho na avaliação do equilíbrio estático e dinâmico se mostrou inversamente proporcional ao avanço da idade, com diferenças significativas $(p<0,001)$ entre idosas sexagenárias (60-64 anos) e septuagenárias (75-79 anos).

Quando estratificadas por faixas de escolaridade, a análise estatística não apontou diferenças significativas para as variáveis estudadas. Contudo, observou-se que idosas analfabetas apresentaram, em todos os testes, índices de aproveitamento mais baixos (Tabela 3):

Com o objetivo de estimar implicações das FE sobre o desempenho nos testes de marcha e equilíbrio corporal foi aplicada a análise de regressão linear múltipla (Tabela 4).A ordem de inserção das variáveis seguiu o modelo forward, do maior para o menor, respeitando a magnitude do coeficiente da correlação de Spearman. Os achados revelaram que quanto maior o déficit cognitivo, mais tempo foi requerido para a execução de tarefas da marcha.

No primeiro modelo ajustado construído apenas para marcha sob tarefa simples (TUGs), o MEEM, a FV-Categoria e a FV-Troca previram significativamente o déficit da velocidade $(p<0,001)$. Os resultados explicaram a variância em $36,4 \%$, $25,7 \%$ e $23,7 \%$, respectivamente. O segundo modelo ajustado foi construído para marcha, associada com a tarefa motora (TUGm). Neste a FV-Intervalo que avaliou a velocidade de raciocínio e a FV-Agrupamento que examinou a capacidade para construção de grupamentos fonéticos se apresentaram como preditoras da baixa do padrão da marcha, explicando a variância em $63 \%$ e $41 \%$, respectivamente $(p<0,001)$.A FV-Categoria foi fator preditor significativo $(p<0,001)$ do modelo ajustado para a velocidade da marcha,associada à tarefa cognitiva (TUGc). Já as subcategorias FVAgrupamento e FV-Categoria previram significativamente $(p<0,001)$ o risco de queda tanto no teste de marcha com ênfase na solução de tarefas funcionais (DGI), como no teste de equilíbrio estático e dinâmico (TEC).

\begin{tabular}{|c|c|c|c|c|c|}
\hline \multirow[t]{2}{*}{ Variáveis } & $\begin{array}{c}60-64 \text { anos } \\
(n=27)\end{array}$ & $\begin{array}{c}65-69 \text { anos } \\
(n=24)\end{array}$ & $\begin{array}{c}70-74 \text { anos } \\
(n=16)\end{array}$ & $\begin{array}{c}75-79 \text { anos } \\
(n=9)\end{array}$ & p \\
\hline & Média/DP & Média/DP & Média/DP & Média/DP & \\
\hline Idade & $61,91 \pm 4,92$ & $66,92 \pm 1,35$ & $72,13 \pm 1,15$ & $76,11 \pm 1,05$ & 0,000 \\
\hline Escolaridade(anos) & $7.18 \pm 3.38$ & $9.00 \pm 3.41$ & $6.20 \pm 4.14$ & $5,25 \pm 3.28$ & 0.172 \\
\hline IMC $\left(\mathrm{kg} / \mathrm{m}^{2}\right)$ & $29.60 \pm 4.92$ & $29.46 \pm 5.28$ & $29.06 \pm 4.44$ & $26.53 \pm 3.93$ & 0.412 \\
\hline Medicamentos & $2,40 \pm 1,76$ & $2,39 \pm 2,10$ & $3,06 \pm 1,70$ & $3,75 \pm 1,49$ & 0,219 \\
\hline Quedas & $1,57 \pm 0,51$ & $1,67 \pm 0,48$ & $1,67 \pm 0,48$ & $1,56 \pm 0,52$ & 0,786 \\
\hline
\end{tabular}




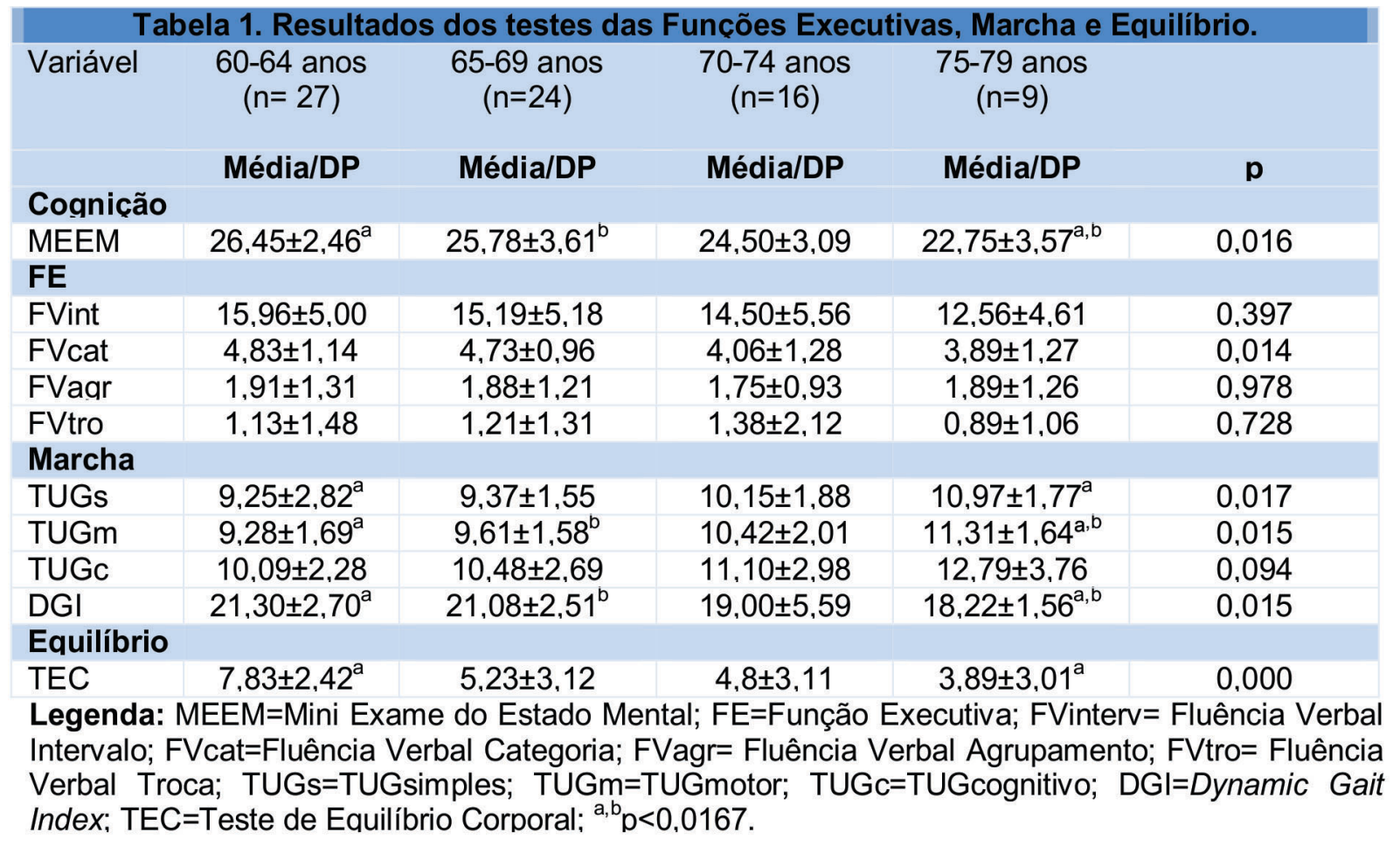

\begin{tabular}{|c|c|c|c|c|}
\hline \multirow[t]{2}{*}{ Variáveis } & $\begin{array}{l}\text { Analfabetos } \\
\qquad(n=2)\end{array}$ & $\begin{array}{c}\text { De } 1-4 \text { anos } \\
(n=24)\end{array}$ & $\begin{array}{c}5 \text { anos ou } \\
+(n=48)\end{array}$ & \\
\hline & Média/DP & Média/DP & Média/DP & p \\
\hline Idade & $66,00 \pm 8,48$ & $67,63 \pm 4,87$ & $67,65 \pm 5,11$ & 0,950 \\
\hline MEEM & $23,50 \pm 0,70$ & $26,78 \pm 2,53$ & $27,54 \pm 3,46$ & 0,237 \\
\hline FVcat. & $3.50 \pm 0.70$ & $4,63 \pm 1,06$ & $4.50 \pm 1,20$ & 0.415 \\
\hline FVint & $7,50 \pm 2,12$ & $15,96 \pm 4,66$ & $14,77 \pm 5,24$ & 0,740 \\
\hline FVagr. & $0,50 \pm 0,70$ & $1,83 \pm 0,90$ & $1,97 \pm 1,27$ & 0,237 \\
\hline FVtro & $0,00 \pm 0,00$ & $1,13 \pm 1,90$ & $1,06 \pm 1,35$ & 0,614 \\
\hline TUGs & $10.50 \pm 0.20$ & $9.64 \pm 1.66$ & $9.69 \pm 1.91$ & 0.816 \\
\hline TUGm & $11,50 \pm 2,52$ & $9,81 \pm 1,59$ & $9,86 \pm 1,90$ & 0.442 \\
\hline TUGc & $11,62 \pm 0,12$ & $10,95 \pm 3,40$ & $10,64 \pm 2,62$ & 0,837 \\
\hline DGI & $19,50 \pm 0,70$ & $20,75 \pm 2,23$ & $20,90 \pm 4,10$ & 0,774 \\
\hline TEC & $5.50 \pm 3.54$ & $5.46 \pm 3.77$ & $5.81 \pm 3.08$ & 0.911 \\
\hline
\end{tabular}

Legenda: MEEM=Mini-Exame do Estado Mental; FVint=Fluência Verbal Intervalo; FVcat=Fluência Verbal Categoria; FVagr=Fluência Verbal Agrupamento; FVtro= Fluência Verbal Troca; TUGs=TUGsimples; TUGm=TUGmotor; TUGc=TUGcognitivo; DGI=Dynamic Gait Index; TEC=Teste de Equilíbrio Corporal; $p<0,0167$.

\section{DISCUSSÃO}

Os achados do presente estudo corroboraram com investigações anteriores ${ }^{1,19,28}$, afirmando que déficits cognitivos aumentam a probabilidade de queda de indivíduos idosos. O caso sobrevém do conjunto de transformações progressivas, as quais atingem o componente cognitivo e consciente humano, tornando-os mais suscetíveis àdéficits ${ }^{12}, 22$. Como consequência disso aumenta a dificuldade da pessoa idosa para compensar o controle sensório-motor tanto durante 


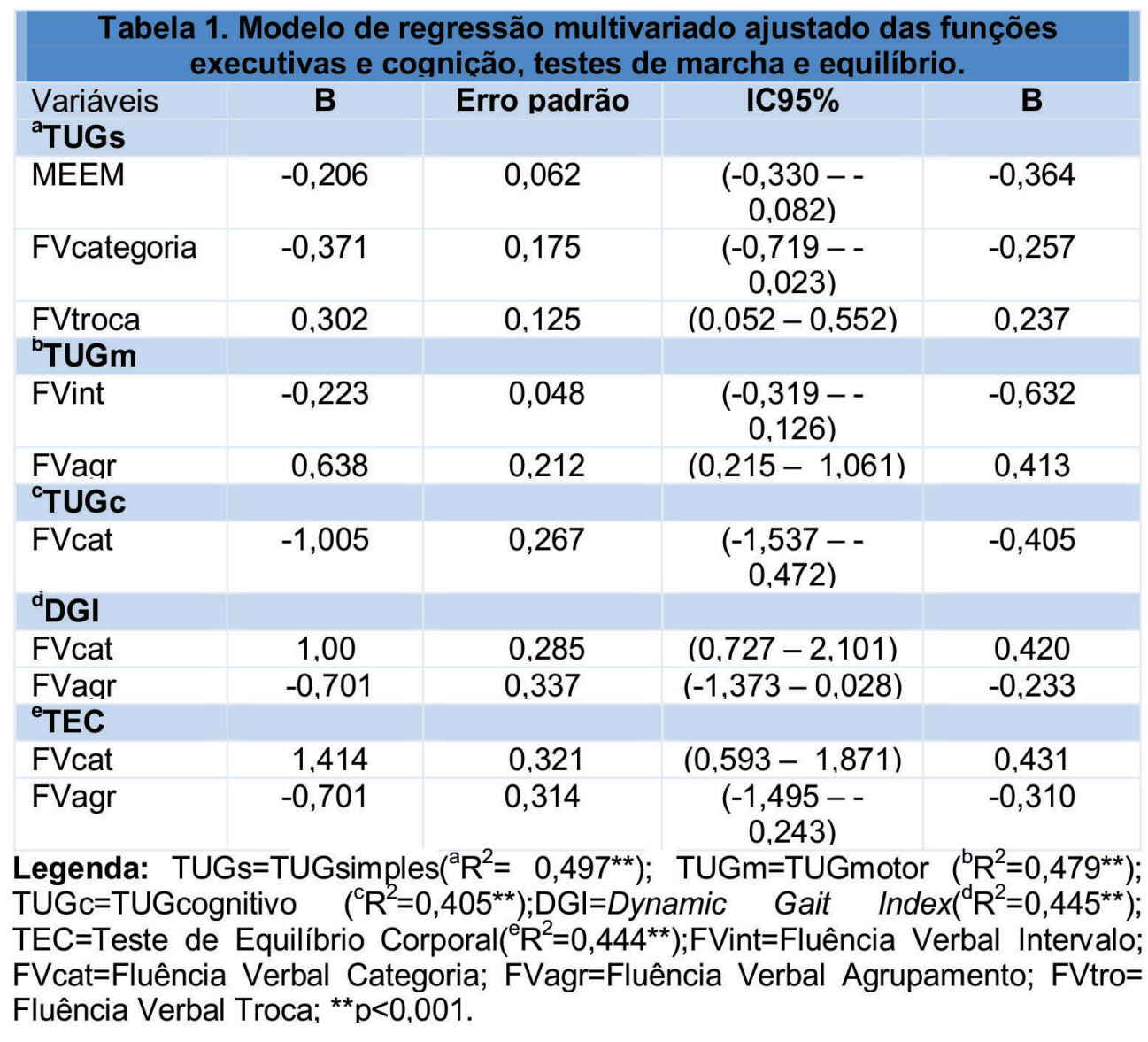

a marcha, como junto à recepção e integração de estímulos sensoriais envolvidos no planejamento e execução de movimentos responsáveis por contrações musculares, as quais são necessárias ao reposicionamento do centro de gravidade sobre a base de sustentação $0^{2,8}$.

No presente estudo, o déficit das FE foi avaliado pelo instrumento $\mathrm{MEEM}^{23}$ e o teste de $\mathrm{FV}^{17,18}$. O MEEM consiste em um teste amplamente aplicado para verificar déficits cognitivos globais, considerando um conjunto de domínios. De acordo com seus pontos de corte e, em consonância com os anos de escolaridade das participantes, não foram observados déficit cognitivos graves. Observou-se,contudo, leve decréscimo do desempenho na avaliação da FV, com o avanço da idade, em especial sobre a velocidade e flexibilidade cognitiva dos avaliados. Corroborando com estudos anteriores ${ }^{12,15}$, um importante achado do presente estudo incidiu na relação entre o déficit cognitivo e o decréscimo do desempenho nos testes de marcha e controle postural, principalmente, entre os participantes deste estudo, em idade avançada.

A associação entre a disfunção executiva e o risco de queda em 188 indivíduos (e"65 anos) em sujeitos com baixo desempenho nos testes de FE apresentou, predominantemente, risco para queda. Por outro lado, os autores também observaram que em indivíduos classificados com a capacidade cognitiva fraca, o risco de queda foi explicado sobretudo por fatores físicos. E que esses estariam relacionados à comprometidos do sistema motor, especialmente, junto ao equilíbrio corporal ${ }^{22}$. No presente estudo, todas as idosas avaliadas eram praticantes regulares de exercícios físicos. De acordo com as avaliações, comprometimentos no desempenho de testes físicos foram observados apenas entre as integrantes do grupo em idade e"75 anos. Este resultado pode ser considerado como 
uma consequência natural do processo do envelhecimento humano'.

De acordo com investigações anteriores, em se tratando do desempenho cognitivo de idosos, a construção de agrupamentos e trocas depende tanto do processamento da memória verbal que está relacionada ao lobo temporal, como do lobo frontal, responsável pelos procedimentos de troca. Sendo esta determinante à eficácia da flexibilidade cognitiva ${ }^{10,15,20}$. Na comparação entre grupos, observou-se que indivíduos acima dos 75 anos, além de apresentarem médias de aproveitamento mais baixas nos testes motores, também exibiram menores índices de desempenho para o tempo de raciocínio, capacidade de busca de nomes de animais e trocas fonéticas, indicando assim maior risco para quedas.

Sabe-se que o componente cognitivo e consciente do idoso para controlar tarefas durante a marcha é mais exigidodo que em indivíduos jovens e adultos ${ }^{18}$. Isso significa dizer que a pessoa idosanecessita de uma boa capacidade cognitiva paraa compensação dedéficits sensóriomotore ${ }^{10}$. Em uma amostra com 737 idosos (e"65 anos) livres deDemência, examinaram a associação entre as FE e a diferença do desempenho foi examinada sob a condição da marcha em tarefa complexa versus simples, utilizaram o teste de realização de trilhas (TMT) e o Mini-Mental State Examination (MEEM). Os autores observaram quea velocidade da marcha em tarefas de caminhada complexa foi predominantemente menor entre os participantes que apresentarambaixo desempenho na avaliação das FE. Também que o declínio percentual da velocidade da marcha foi menor, a depender do tipo da tarefa. Entre as explicações para o caso, os autores citam o avanço da idadecomo principal responsável pelo declínio do sempenho das FE, sobretudo, em indivíduos com atrofia no lobo temporal, área pré-frontal ${ }^{20}$.

A literatura especializada considera para a população idosos quea atrofia do corpo caloso seja correlato à desaceleração da marcha ${ }^{29}$. Isso significa dizer que a avaliação da velocidade da marcha, em tempo normal, consiste em fator preditor de comprometimentos da capacidade cognitiva ${ }^{20,29}$. Sendo assim, é recomendadoa estimulação constante dessas áreasem atividades com idosos.Diante disso, estudos detalharam os benefícios da prática regular do exercício físico em condições de tarefa dupla e múltipla à prevenção de quedas ${ }^{4,14,15}$. Essa técnica se apresenta eficaz, uma vez que seus exercícios auxiliam no treinamento da capacidade de atenção, memória e raciocínio da pessoa, reduzindo assim o risco de queda ${ }^{21}$.

Corroborando com estudos anteriores ${ }^{22,23}$, os resultado do presente estudoindicaram relação entre os anos de escolaridade eo déficit do desempenho nos testes de FV. O desfecho também foi seguido pelo baixo padrão da marcha e o déficit do controle do equilíbrio. Partindo para a análise de regressão multivariada foi observado que o desempenhodas idosas nos testes de marcha e equilíbrio se apresentou sucetíveis à interferência das FE. Os modelos ajustados demonstraram existir influência do déficit cognitivo entre $23 \%$ e $63 \%$ sobre o padrão da marcha e entre $31 \%$ a $43 \%$ sobre o controle do equilíbrio estático e dinâmico. Essesresultados alertarampara a baixa do dempenho cognitiva como fator associado ao risco de queda.Salienta-se, todavia, que os resultados dos testes físicos não advertiram para o risco de queda entreidosasna faixa etária, entre60 e74 anos. Por outro lado, considerandoa associação doexame das FE com os testes físicosfoi obtido risco relativode queda para toda a população.Isso significa dizer que os testes utilizadossão complementares, sugerindo-se sua aplicação de forma conjunto, uma vez que o evento da queda é multifatorial ${ }^{1,13,14}$.

Admite-se que tanto a lentidão psicomotora da fala de algumas idosas, como o nível de escolaridadepossamter reduzidoseudesempenho nos testes deFV. No entanto, isso não significaque o processamento de resgate de palavras e léxico semântico desta população esteve comprometido ${ }^{18}$. Assim, sabendo-se que a diagnóse precisa do déficitdas FE só é possível mediante testes clínicos, após a conclusão dopresente estudo,idosasque apresentaram baixoaproveitamento na avaliação das FEforam encaminhados a um profissional específico da área clínica.Considera-se como uma possível limitação do presente estudo a falta de grupo controle, o que impossibilitou a verificação 
de associações de causalidade entre as variáveis investigadas.

\section{CONCLUSÃO}

De forma geral, observou-se influência das FE sobre o processamento neural necessário ao conjunto de ajustes do controle da marcha e do equilíbrio corporal. Constatou-se que déficits nas FE aumentam a probabilidade de queda de mulheres idosas fisicamente ativas, em especial, aquelas com baixa escolaridade e/ou em idade superior a 75 anos. A partir disso, aconselha-se como medida à prevenção de queda que idosos participem semanalmente de programas de exercícios físicos, além de que os treinamentos incluam atividades que promovam a capacidade cognitiva, de atenção e memória dos idosos, principalmente, em situações de tarefa dupla e múltipla. Por fim, sugere-se a realização de futuros estudos à avaliação do risco de quedas de mulheres idosas praticantes regulares de exercícios físicos. Contudo que os mesmos incluam grupo controle e contemplem o exame do desempenho físico e cognitivo. Pois isso ampliará e qualificarão rastreamento de fatores associados ao risco de quedas nessa população.

\section{AGRADECIMENTOS}

Ao Programa de Iniciação Cientifica (PIBIC/ 215-2016) da Universidade Federal do Vale do São Francisco e o CNPq pela cessão de duas bolsas à realização deste estudo.

\section{REFERÊNCIAS}

1. Callisaya ML, Blizzard L, Schmidt MD, Martin KL, MacGinley JL, Sanders LM et al. Gait, gait variability and the risk of multiple incident falls in older people: A population-based study. Age Ageing. 2011; 40(4):481487.

2. Tinetti ME. Performance-oriented assessment of mobility problems in elderly patients. J Am Geriatr Soc. 1986; (34):119-126.

3. Perracini MR. Prevenção e manejo de quedas no Idoso. Prevalência de quedas em idosos institucionalizados no Lar das Vovozinhas e Lar dos Vovozinhos da cidade de Londrina. http://www.portalsaudebrasil.com/ artigospsb/idoso092.pdf. Published 2009.

4. Amboni M, Barone P HJM. Cognitive contributions to gait and falls: Evidence and implications. Mov Disord. 2013; 28(11):1520-1533.

5. Instituto Brasileiro de Geografia e Estatística (IBGE). Censo demográfico 2010. 6. Freiberger E, de Vreede P. Falls recall-limitations of the most used inclusion criteria. Eur Rev Aging Phys Act. 2011; 8(2):105-108.

7. Rowe JW, Kahn RL. Successful Aging 2.0: Conceptual Expansions for the 21st Century. Journals Gerontol Ser B Psychol Sci Soc Sci. 2015; 70(4):593-596.

8. Graafmans WC, Ooms ME, Hofstee HMA, Bezemer PD, Bouter LM, Lips P. Falls in the Elderly: A Prospective Study of Risk Factors and Risk Profiles. Am J Epidemiol. 1996; 143(11):1129-1136.

9. Yuan J, Blumen HM, Verghese J, Holtzer R. Functional connectivity associated with gait velocity during walking and walking-while-talking in aging: A resting-state fMRI study. Hum Brain Mapp. 2015; 36(4):1484-1493.
10. Sheridan PL, Solomont J, Kowall N, Hausdorff JM. Influence of Executive Function on Locomotor Function: Divided Attention Increases Gait Variability in Alzheimer's Disease. J Am Geriatr Soc. 2003; 51(11):1633-1637.

11. Kirkwood RN. Biomecânica da marcha em idosos caidores e não caidores/ : uma revisão da literatura. Rev Bras Ciência e Mov Hum. 2006; 14(4):103-110.

12. Duarte M, Freitas SM. Revision of posturography based on force plate for balance evaluation. Rev Bras Fisioter. 2010; 14(3):183-192.

13. Simoceli L, Bittar RMS, Bottino MA, Bento RF. Perfil diagnóstico do idoso portador de desequilíbrio corporal: resultados preliminares. Rev Bras Otorrinolaringol. 2003; 69(6):772-777.

14. Zijlstra A, Ufkes T, Skelton DA, Lundin-Olsson L, Zijlstra W. Do dual tasks have an added value over single tasks for balance assessment in fall prevention programs? A mini-review. Gerontology. 2008; 54(1):40-49.

15. Andrade LP, Stella F, Barbieri FA, Rinaldi NM, Hamanaka AYY, Gobbi LTB. Efeitos de tarefas cognitivas no controle postural de idosos: Uma revisâo sistemática. Motricidade. 2011; 7(3):19-28.

16. Argimon I, Bicca M, Timm L, Vivan A. Funções executivas e a avaliação de flexibilidade de pensamento em idosos. Rev Bras Ciências do Envelhec Hum. 2006; 3(2):35-42.

17. Da Silva TBL, Yassuda MS, Guimarães VV, Florindo AA. Fluência verbal e variáveis sociodemográficas no processo de envelhecimento: Um estudo epidemiológico. Psicol Reflexão e Crítica. 2011; 24(4):739-746. 
18. Magila C, Caramelli P. Funções executivas no idoso. In: O. V. Forlenza \& P. Caramelli (Eds.), Neurosiquiatria Geriátrica (p. 517-525). São Paulo: SP, Ateneu; 2001.

19. Menezes RL De, Bachion MM. Estudo da presença de fatores de riscos intrínsecos para quedas, em idosos institucionalizados. Cien Saude Colet. 2008; 13(4):12091218.

20. Coppin AK, Shumway-Cook A, Saczynski JS, Patel KV, Ble A, Ferrucci L. et al. Association of executive function and performance of dual-task physical tests among older adults: Analyses from the InChianti study. Age Ageing. 2006; 35(6):619-624.

21. Erikson KI, Voss MW, Prakasch RS, Basak C, Szabo $A C L$, Chaddock L. et al. Exercise training increases size of hippocampus and improves memory. Proc Natl Acad Sci. 2011; 108(7):3017-3022.

22. Buracchio TJ, Mattek NC, Dodge HH, Hayes TL, Pavel $\mathrm{M}$, Howieson DB KJ. Executive function predicts risk of falls in older adults without balance impairment. BMC Geriatr. 2011; 11(74):2-7.

23. Bertolucci PH, Brucki SM, Campacci SR, Juliano Y. O Mini-Exame do Estado Mental em uma população geral. Impacto da escolaridade. Arq Neuropsiquiatr. 1994; 52(1):1-7.

24. Podsiadlo D, Richardson S. The time up and go, a test of basic functional mobility for frail elderly persons. J Am Geriatr Soc. 1991; 39(2):142-148.
25. De Castro SM, Perracini MR, Ganança FF. Dynamic gait index-Brazilian version. Braz J Otorhinolaryngol. 2006; 72(6):817-825.

26. Nascimento MM, Appell IC, Appell HJ. Teste de equilíbrio corporal (TEC) para idosos independentes. Rev Port Ciências do Desporto. 2012; 12(2):71-81.

27. WHO. Physical status: the use and interpretation of anthropometry. Report of a WHO Expert Committee. World Health Organ Tech Rep Ser. 1995; 854:1-452.

28. Shumway-Cook A, Brauer S, Woollacott M. Predicting the Probability for Falls in Community-Dwelling Older Adults Using the Timed Up \& Go Test. 2000; 80(9):896903.

29. Deshpande N, Metter EJ, Bandinelli S, Guralnik J, Ferrucci L. Gait speed under varied challenges and cognitive decline in older persons: A prospective study. Age Ageing. 2009; 38(5):509-514.

\section{Correspondência}

Marcelo de Maio Nascimento

Av. José de Sá Maniçoba S/N, Petrolina. Universidade Federal do Vale do São Francisco

CEP: $56304-917$

Petrolina - Pernambuco - Brasil.

E-mail: marcelo.nascimento@univasf.edu.br 